04.2

\title{
Исследования альфвеновских мод на токамаке Глобус-М2 с использованием многочастотного допплеровского рефлектометра $V$-диапазона
}

\author{
(C) В.В. Буланин ${ }^{1,2}$, И.М. Балаченков ${ }^{2}$, В.И. Варфоломеев ${ }^{2}$, В.К. Гусев ${ }^{2}$, Г.С. Курскиев ${ }^{2}$, В.Б. Минаев ${ }^{2}$, \\ М.И. Патров ${ }^{2}$, А.В. Петров ${ }^{1}$, Ю.В. Петров ${ }^{2}$, А.М. Пономаренко $^{1}$, А.Ю. Тельнова ${ }^{2}$, П.Б. Щеголев ${ }^{2}$, \\ А.Ю. Яшин ${ }^{1,2}$
}

\author{
${ }^{1}$ Санкт-Петербургский политехнический университет Петра Великого, Санкт-Петербург, Россия \\ 2 Физико-технический институт им. А.Ф. Иофрфе РАН, Санкт-Петербург, Россия \\ E-mail: V.Bulanin@spbstu.ru
}

Поступило в Редакцию 14 октября 2020 г.

В окончательной редакции 11 ноября 2020г.

Принято к публикации 11 ноября 2020 г.

\begin{abstract}
Приведены новые сведения о развитии альфвеновских мод в сферическом токамаке Глобус-М2. Данные получены с использованием допплеровского рефлектометра $V$-диапазона с частотами зондирующего излучения от 50 до $75 \mathrm{GHz}$. В качестве многочастотного источника зондирующего излучения использовался синтезатор СВЧ-гармоник. В результате использования нового рефлектометра определена локализация тороидальной альфвеновской моды при магнитном поле 0.5 T. Зарегистрированы спектральные компоненты альфвеновской моды с допплеровскими сдвигами частоты, обусловленные тороидальным вращением плазмы. В центральных областях разряда исследованы так называемые альфвеновские каскады.
\end{abstract}

Ключевые слова: плазма, токамак, альфвеновские моды, допплеровское обратное рассеяние.

DOI: 10.21883/PJTF.2021.04.50645.18584

В экспериментах на токамаке Глобус-М для регистрации альфвеновских мод впервые был предложен и реализован метод допплеровского обратного рассеяния (ДОР, DBS) [1-3]. Диагностика методом ДОР была основана на регистрации колебаний скорости дрейфа в скрещенных радиальном электрическом поле альфвеновской волны и магнитном поле токамака [3]. Измерения, проведенные с предельной частотой зондирования $48 \mathrm{GHz}$, показали, что для восстановления пространственного распределения обнаруженной тороидальной альфвеновской моды (ТАЕ) требуется использовать для зондирования излучение более высоких частот. Целью настоящей работы является исследование альфвеновских мод с допплеровского рефлектометра с частотами зондирования от 50 до $75 \mathrm{GHz}$, находящимися в более высокочастотном $V$-диапазоне, который соответствует расположению области обратного рассеяния в центральной зоне токамака. Эксперименты проводились на реконструированном сферическом токамаке Глобус-М2, в котором сохранена вакуумная камера, а магнитная система усилена с целью увеличения тороидального магнитного поля до $1 \mathrm{~T}$ и тока плазмы до 0.5 МА [4]. Микроволновая схема была аналогична схеме, предложенной впервые на токамаке DIII-D [5]. Использовались умножитель частоты в качестве синтезатора зондирующего излучения на шести частотах $(50,55,60,65,70,75 \mathrm{GHz})$ и местный гетеродин с частотой $63 \mathrm{GHz}$. Применялся набор полосовых фильтров, который обеспечивал селекцию сигналов на шести промежуточных частотах. Квадратурное детектирование этих сигналов позволяло получать шесть I (in-phase) и
Q (quadrature) сигналов, которые поступали на многоканальный аналого-цифровой преобразователь LTR210 с частотой оцифровки $5 \mathrm{MHz}$. В целом микроволновая схема была изготовлена в DOC company SPB. Зондирование плазмы и прием обратно рассеянного излучения О-моды осуществлялись через две раздельные рупорные антенны. Система поворота антенн, находящаяся в экваториальной плоскости на внешнем обходе тора, позволяла осуществлять наклон как в тороидальном, так и в полоидальном направлении. Выходные сигналы IQ-детекторов использовались для определения допплеровского частотного сдвига и исследования колебаний скоростей вращения плазмы на частоте альфвеновских колебаний. Допплеровский сдвиг определялся как производная фазы комплексного сигнала IQ-детектора, а колебания производной фазы на частотах альфвеновских колебаний выделялись при спектральном анализе [3].

Первые эксперименты с использованием многочастотной диагностики допплеровского обратного рассеяния были ориентированы на выяснение того, насколько TAE-мода, обнаруженная ранее на токамаке Глобус-М при тороидальном магнитном поле $0.5 \mathrm{~T}$ [2], простирается внутрь разряда. На рис. 1 отмечен фрагмент радиального профиля амплитуды полоидальной компоненты магнитного поля ТАЕ, полученный в разряде \#37001 при частотах зондирования 20, 29, 39 и $48 \mathrm{GHz}$. На токамаке Глобус-М2 в разряде \#39197 были воспроизведены условия разряда \#37001 (а именно магнитное поле $0.5 \mathrm{~T}$, ток разряда $240 \mathrm{kA}$ и средняя по хорде плотность плазмы $4 \cdot 10^{19} \mathrm{~m}^{-3}$ ) при той же мощности и энергии 


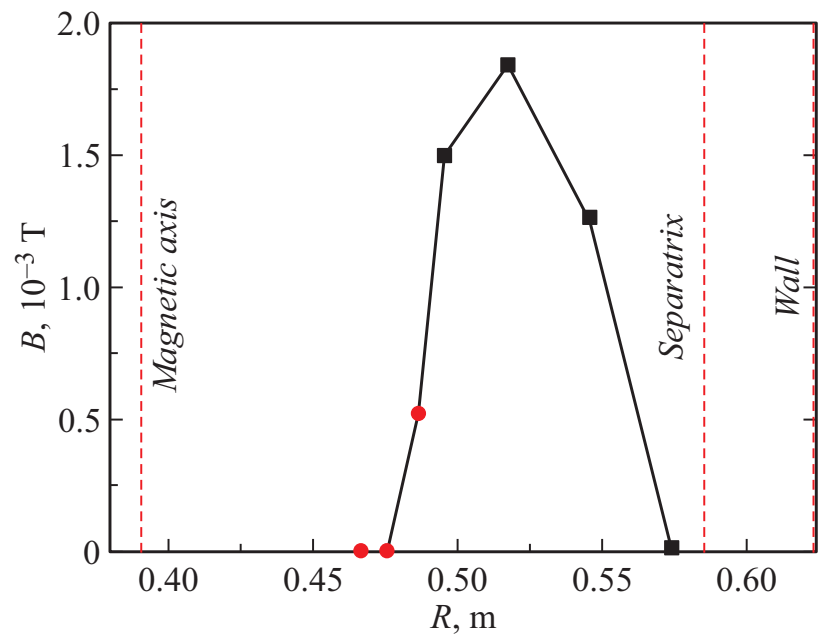

Рис. 1. Радиальная зависимость амплитуды колебаний полоидальной компоненты магнитного поля в ТАЕ. Квадраты $\# 37001, t=145 \mathrm{~ms}$, кружки - \#39197, $t=137 \mathrm{~ms}$.

инжектируемого пучка нейтральных атомов $(800 \mathrm{~kW}$ и $28 \mathrm{kV}$ соответственно). В результате в разряде \#39197 развивалась альфвеновская мода с той же частотой и амплитудой, что и в разряде \#37001. Сравнение проводилось по измерениям с помощью магнитных зондов (МР). В разряде \#39197 использовался новый многочастотный допплеровский рефлектометр. Плотность плазмы была такова, что микроволновые пучки на частотах 75,70 и $65 \mathrm{GHz}$ проходили объем плазмы практически без искривления траектории, когда условия ДОР не обеспечивались. Данные, полученные с использованием излучения на частотах 60, 55 и $50 \mathrm{GHz}$, представлены на рис. 1 в виде фрагмента радиальной зависимости амплитуд колебаний полоидального магнитного поля на частотах TAE. Можно полагать, что данные двух экспериментов описывают область локализации ТАЕ в целом. Полученное радиальное распределение убедительно демонстрирует спад колебаний ТАЕ к центру разряда и их исчезновение при нормированном малом радиусе токамака $\rho=0.5$. Благодаря вновь полученным данным мы смогли отобрать из предложенных ранее теоретических моделей, описывающих развитие ТАЕ в токамаке Глобус-М/M2 [6], ту, которая дает наиболее близкие к эксперименту результаты по частоте и локализации моды. Эта модель плазмы с закрепленной границей из работы [6] и будет в дальнейшем использоваться для моделирования ТАЕ в токамаке Глобус-М2.

При увеличении магнитного поля до $0.7 \mathrm{~T}$ в токамаке Глобус-М2 с использованием магнитной диагностики и новой схемы ДОР были обнаружены ТАЕ-колебания в диапазоне $300 \mathrm{kHz}$. Отметим, что эта мода развивалась на поздней стадии нагрева плазмы с помощью инжекции пучка нейтральных атомов (NBI), через $35 \mathrm{~ms}$ после включения NBI. Характерные спектры колебаний скорости и сигналов магнитного зонда приведены на рис. 2. На рисунке видны спектральные составляющие, отстоящие друг от друга на расстояние $48 \mathrm{kHz}$. Путем анализа сигналов массива магнитных зондов было установлено, что эти частотные составляющие соответствуют альфвеновским модам с тороидальными числами $n=1,2$ и 3. Такие спектры регистрировались методом ДОР при частотах зондирования 50 и $55 \mathrm{GHz}$ и не наблюдались на частотах 39 и $60 \mathrm{GHz}$, что, видимо, соответствует существованию локального максимума в окрестности нормированного радиуса $\rho=0.5$. Аналогичные спектры наблюдались ранее на токамаках DIII-D [7], JET [8], COMPASS [9] и NSTX [10]. Они объяснялись тем, что частота колебаний, наблюдаемая в лабораторной системе координат, сдвинута относительно альфвеновской частоты $f_{\text {TАE }}$ на допплеровский частотный сдвиг, который обусловлен тороидальным вращением плазмы, вызываемым продольной инжекцией пучка нейтральных атомов [7]:

$$
f_{n}^{\mathrm{LAB}}=f_{\mathrm{TAE}}+n f_{T}(r) .
$$

Здесь $f_{T}(r)=V_{T}(r) / 2 \pi R\left(V_{T}-\right.$ тороидальная скорость вращения плазмы). Эта спектральная зависимость от модового числа $n$ качественным образом отличается от тех зависимостей, которые отмечались на токамаке Глобус-М в периферийных областях разряда, когда модовым числам $n$ соответствовали кратные спектральные гармоники альфвеновской частоты: $f_{n}^{\mathrm{LAB}}=n f_{\mathrm{TAE}}$ [3]. Можно полагать что отличие в спектрах обусловлено тем, что в при больших магнитных полях $B_{T}=0.7 \mathrm{~T}$, когда обеспечивается лучшее удержание ионов высоких энергий, через определенное время после включения NBI скорость тороидального вращения, определенная по допплеровскому сдвигу $f_{T}(r)$, достигает значения,

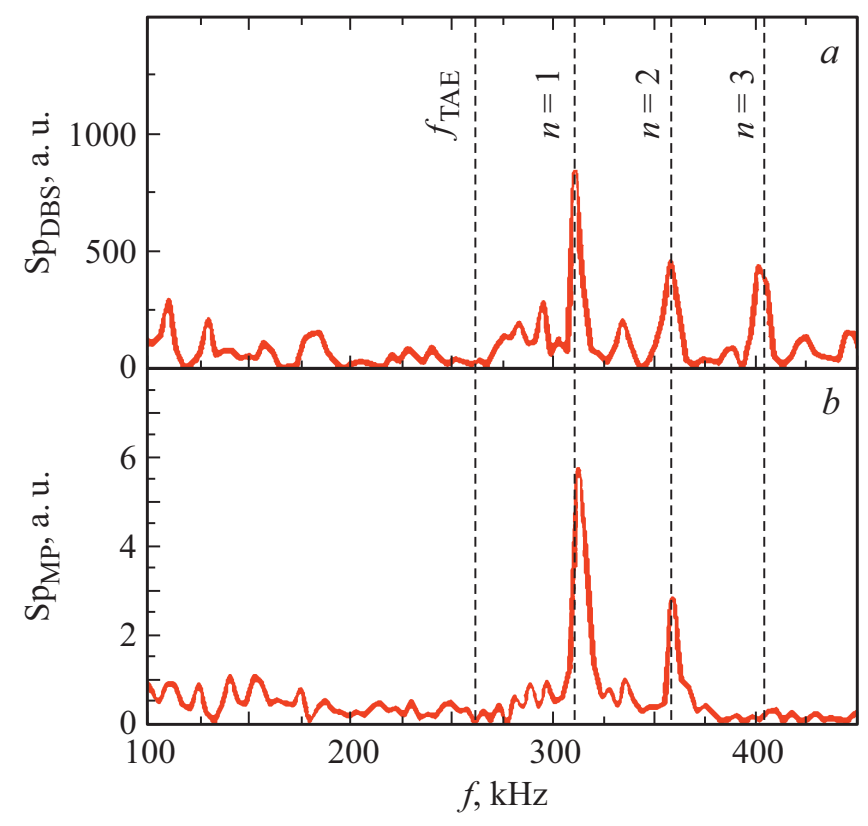

Рис. 2. $a-$ спектр колебаний скорости вращения плазмы в разряде \#39232, частота зондирования $50 \mathrm{GHz} ; b-$ спектр колебаний сигналов магнитного зонда. Время $159.7 \mathrm{~ms}$. 


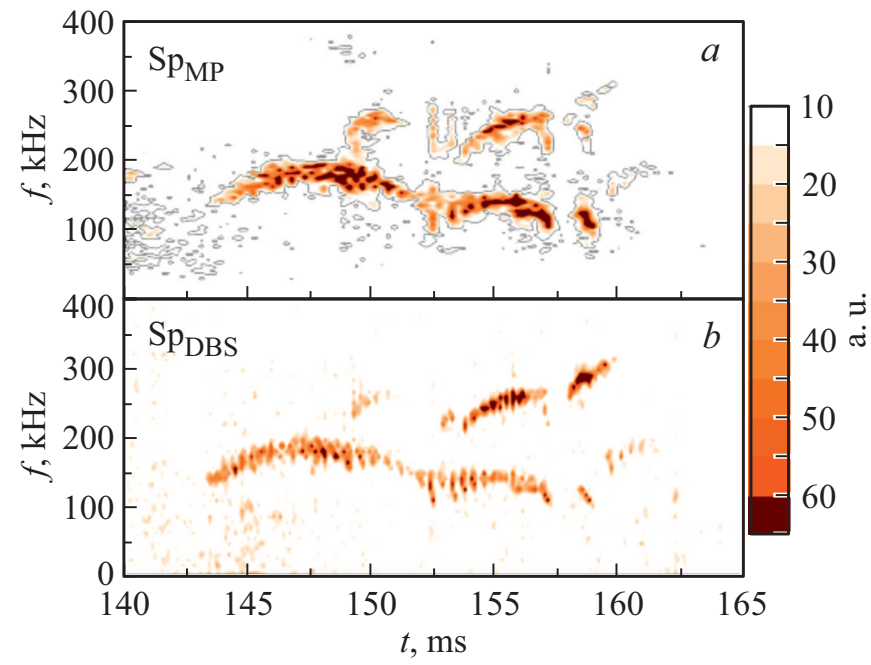

Рис. 3. Спектрограммы колебаний сигнала магнитного зонда $(a)$ и скорости вращения $(b)$ при возникновении альфвеновских каскадов в разряде \#38388 при зондировании плазмы на частоте $48 \mathrm{GHz}$.

равного $1.6 \cdot 10^{5} \mathrm{~m} / \mathrm{s}$ (для большого радиуса токамака $R=52 \mathrm{~cm}$ ). Эти данные планируется сравнить с результатами измерения тороидальной скорости методом CHERS. Динамику нарастания тороидальной скорости проследить было невозможно, так как альфвеновская мода с допплеровски сдвинутыми частотами возникала только через $35 \mathrm{~ms}$ после начала NBI.

Необходимость использования более высокочастотного диапазона зондирующего излучения в наибольшей степени проявилась при регистрации альфвеновских колебаний в разрядах с обращенным широм магнитного поля (RSAE) или так называемых альфвеновских каскадов. Такие колебания развиваются в области минимума коэффициента запаса устойчивости $q_{\min }$, т. е. в центральных областях разряда, и для их регистрации методом ДОР необходимо в условиях токамака Глобус-М2 переходить на зондирование с использованием излучения $V$-диапазона. Колебания RSAE на токамаке Глобус-М2 были зарегистрированы с помощью магнитных зондов в разрядах в NBI на стадии нарастания тока (рис. 3, $a$ ), когда в плазме возможно появление обратного шира магнитного поля [11]. Возникновение RSAE объяснялось уменьшением параметра $\beta_{e}=8 \pi p_{e} / B_{0}^{2}\left(p_{e}-\right.$ газокинетическое давление электронов, $B_{0}$ - поле на магнитной оси) при увеличении магнитного поля в токамаке Глобус-М2 до 0.7 T [11]. Одновременно с появлением колебаний RSAE в сигналах магнитного зонда колебания с такой же эволюцией частоты наблюдались в спектрограммах колебаний скорости, которая определялась методом ДОР (рис. $3, b$ ). В каскаде наблюдались подъем частоты при уменьшении $q_{\min }$, отвечающий развитию RSAE, и спад частоты при переходе к колебаниям TAE. Детальный анализ спектров, позволяющий определить минимальные значения $q$, приведен в работе [11]. Отме- тим, что привлечение данных ДОР о локализации RSAE позволяет оценить радиус с минимальным значением $q$ и сравнить этот радиус с расчетными данными, полученными с привлечением кода ASTRA [11].

Таким образом, эксперименты с использованием допплеровского обратного рассеяния в более высокочастотном $V$-диапазоне обеспечили измерения радиальных профилей интенсивности различных альфвеновских мод, возникающих при нейтральной инжекции, вдоль всего малого радиуса установки. Это позволило окончательно определить локализацию ТАЕ при магнитном поле $0.5 \mathrm{~T}$, что способствовало выбору правильной модели для расчета альфвеновского континуума и структуры мод из ранее предложенных [6]; применить метод магнитогидродинамической спектроскопии для определения эволюции $q_{\min }$ в токамаке Глобус-М2 при регистрации альфвеновских каскадов; с помощью анализа спектров определить скорость тороидального вращения плазмы в области одновременного развития ТАЕ с различными волновыми числами $n$.

\section{Финансирование работы}

Работа выполнена при поддержке Российского научного фонда (проект № 17-12-01177-П).

\section{Конфликт интересов}

Авторы заявляют, что у них нет конфликта интересов.

\section{Список литературы}

[1] В.В. Буланин, В.К. Гусев, Г.С. Курскиев, В.Б. Минаев, М.И. Патров, А.В. Петров, М.А. Петров, Ю.В. Петров, А.Ю. Тельнова, А.Ю. Яшин, Письма в ЖТФ, 43 (23), 40 (2017). DOI: 10.21883/PJTF.2017.23.45274.16986 [Пер. версия: $10.1134 / \mathrm{S} 1063785017120033]$.

[2] В.В. Буланин, В.К. Гусев, Г.С. Курскиев, В.Б. Минаев, М.И. Патров, А.В. Петров, Ю.В. Петров, А.Ю. Яшин, Письма в ЖТФ, 45 (21), 44 (2019). DOI: 10.21883/PJTF.2019.21.48474.1798 [Пер. версия: 10.1134/S1063785019110051].

[3] В.В. Буланин, Н.Н. Бахарев, В.К. Гусев, Г.С. Курскиев, В.Б. Минаев, М.И. Патров, А.В. Петров, Ю.В. Петров, Н.В. Сахаров, П.Б. Щёголев, А.Ю. Тельнова, С.Ю. Толстяков, А.Ю. Яшин, ВАНТ. Сер. Термоядерный синтез, 42 (4), 52 (2019). DOI: 10.21517/0202-3822-2019-42-4-52-60

[4] N.N. Bakharev, I.M. Balachenkov, F.V. Chernyshev, I.N. Chugunov, V.V. Dyachenko, V.K. Gusev, M.V. Iliasova, E.M. Khilkevitch, N.A. Khromov, E.O. Kiselev, A.N. Konovalov, G.S. Kurskiev, V.B. Minaev, A.D. Melnik, I.V. Miroshnikov, A.N. Novokhatsky, M.I. Patrov, Yu.V. Petrov, N.V. Sakharov, P.B. Shchegolev, A.E. Shevelev, O.M. Skrekel, A.Yu. Telnova, V.A. Tokarev, S.Yu. Tolstyakov, E.A. Tukhmeneva, V.I. Varfolomeev, A.V. Voronin, Plasma Phys. Rep., 46, 675 (2020).

DOI: $10.1134 / \mathrm{S} 1063780 \mathrm{X} 20070016$ 
[5] W.A.Peebles, T.L. Rhodes, J.C. Hillesheim, L. Zeng, C. Wannberg, Rev. Sci. Instrum., 81, 10D902 (2010). DOI: $10.1063 / 1.3464266$

[6] С.Ю. Медведев, А.А. Мартынов, В.К. Гусев, Ю.В. Петров, М.И. Патров, А.Ю. Тельнова, А.А. Иванов, Ю.Ю. Пошехонов, ВАНТ. Сер. Термоядерный синтез, 41 (2), 95 (2018). DOI: $10.21517 / 0202-3822-2018-41-2-95-104$

[7] E.J. Strait, W.W. Heidbrink, A.D. Turnbull, Plasma Phys. Control. Fusion, 36, 1211 (1994).

DOI: $10.1088 / 0741-3335 / 36 / 7 / 008$

[8] S.E. Sharapov, Trans. Fusion Sci. Technol., 61, 104 (2012). DOI: 10.13182/FST12-A13497

[9] T. Markovic, J. Stockel, J. Seidl, A. Melnikov, S. Medvedev, in Proc. of the 23rd Annual Conf. of Doctoral Students (WDS 2014) (Prague, 2014), p. 199-203.

[10] M. Podestá, R.E. Bell, A. Bortolon, N.A. Crocker, D.S. Darrow, A. Diallo, E.D. Fredrickson, G.-Y. Fu, N.N. Gorelenkov, W.W. Heidbrink, G.J. Kramer, S. Kubota, B.P. LeBlanc, S.S. Medley, H. Yuh, Nucl. Fusion 52, 094001 (2012). DOI: 10.1088/0029-5515/52/9/094001

[11] И.М. Балаченков, Ю.В. Петров, В.К. Гусев, Н.Н. Бахарев, В.В. Буланин, В.И. Варфоломеев, Н.С. Жильцов, Е.О. Киселёв, Г.С. Курскиев, В.Б. Минаев, М.И. Патров, А.В. Петров, А.М. Пономаренко, Н.В. Сахаров, А.Ю. Тельнова, В.А. Токарев, Н.А. Хромов, П.Б. Щеголев, А.Ю. Яшин, Письма в ЖТФ, 46 (23), 3 (2020).

DOI: $10.21883 /$ PJTF.2020.23.50338.18450 